\title{
The Design of Automatic Mine Drainage System
}

\author{
Gao Qifeng \\ Zhejiang Industry Polytechnic College \\ ShaoxingChina, 312000 \\ gqf8443235@163.com
}

\author{
Liu Jian \\ Zhejiang Industry Polytechnic College \\ ShaoxingChina, 312000 \\ 201j08@163.com
}

\begin{abstract}
The mine drainage system undertakes an important task in underground water exclusion, while the traditionally manual system, which has many disadvantages, can not adapt to the mine production and management mode of modern enterprises. Based on the reality of coal production and the trend of modern automatic industries, this article designs an automatic mine drainage system, making coal production safety, reliability and efficiency greatly improved.
\end{abstract}

Keywords-Underground; Automatic drainage system; Design

\section{INTRODUCTION}

Underground drainage system is one of the four systems in coal production, undertaking the important task of underground water exclusion. However, the current drainage system is still manual-operating mode in China at present. With the development of modern science and technology, especially that of the microelectronic technology and the control theory which have been applied in all trades, the security, reliability and efficiency of current industrial equipment have been greatly improved.

The traditional drainage system can not adapt to the contemporary administration and operation of modern coal enterprises, which urges a new drainage system to guarantee the safety and efficiency of coal mining. The automatic mine drainage system is an automated system which can satisfy the need.

On the basis of the traditional drainage system, it is the application of modern industrial control technology and detection equipment to realize automatic mine drainage system. It has also extends its function that when pumps unmanned operating underground, the system can choose the best operation scheme in accordance with the specific conditions and requirements, and provide datum for ground monitoring system.

\section{THE COMPOSITION AND CHARACTERISTICS OF UNDERGROUND DRAINAGE SYSTEM}

The mine drainage system mainly consists of drain sump, inflow pipes, pumps, drainpipes, irrigation devices and several control valves.

\section{A. Main features}

1)Water level is subject to various factors, such as the amount of gushing water and drainage in mine, and net volumes and sediment of water warehouses, the control of which should leave sufficient safety margin.
2)The pump motor is of high voltage and big power, and the process of start is relatively complex.

3)The bibulous way of bottomless valve is widely used in water pumps. In the drainage system are water pumps, water pipes and irrigation device. Before start pumps and water pipes should be filled with water.

4)A lot of suspended solids in the gushing water will greatly damage the water pump and easily deposit on the drainage pipes, and that will lessen the area of passage, affecting the pump and drainage efficiency.

5)Under the "peak-valley price" system set by the state power department, work time of the underground drainage should be arranged in "avoiding peak" as far as possible to reduce drainage electric expenses. [1][2]

6)Mine drainage system is designed according to the maximum gushing water and the corresponding quantity of pumps in mine. When pump is in normal state, its back-up ability is relatively high. [1][2]

\section{The Double Logic CONTROL Mode IN WATER PUMP STATION}

It is the simplest control mode to judge the operation states of pump station in accordance with well levels. Realized by microcomputer control system, the double logic control system will obtain the operation state of pump according to the water level setting and logic operation results.

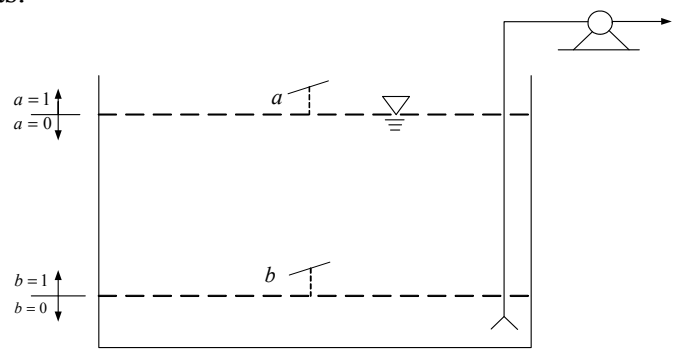

Figure 1. double logic control in water pumping station

As shown in table 1, when the well surface level in water pump room is between level $a$ and $b$, pumps will automatically open and stop along with the change of water level: when the water level is higher than level a, pump starts draining; while when the level is lower than level b, pump stop working. Therefore, the water level switch should be set at the corresponding level in logic control system. When the 
water level is higher than the prescribed value, the water switch close through electricity and the logic value is one; while when the water level is below the prescribed value, the water switch cut off through electricity and the logic value is zero.

Judging from the working process, it is a logical system which can memorize variables through the controller's input or output device. The variables are the water level switch a and $b$; while the additional variable is Pt- 1 which represents the current state of the pump. These three variables can form eight kinds of combinations. According to the given requirements, each combination results should comply with the relational calculus in table I.

TABLE I. LOGIC OPERATION OF VARIABLES

\begin{tabular}{|c|ccc|c|}
\hline Number & a & b & Pt-1 & P \\
\hline 1 & 0 & 0 & 0 & 0 \\
\hline 2 & 0 & 0 & 1 & 0 \\
\hline 3 & 0 & 1 & 0 & 0 \\
\hline 4 & 0 & 1 & 1 & 1 \\
\hline 5 & 1 & 0 & 0 & - \\
\hline 6 & 1 & 0 & 1 & - \\
\hline 7 & 1 & 1 & 0 & 1 \\
\hline 8 & 1 & 1 & 1 & 1 \\
\hline
\end{tabular}

The number 5 and 6 in the table, these two kinds of logical combinations, which do not accord with the actual situation, are breakdowns and will not be considered. Hence establishes a logic operation and follows that:

$$
P=a b+b P_{t-1}=b\left(a+P_{t-1}\right)
$$

As the AC contactor controlling pump operation, the electric connection and disconnection of its coils will be in consistent with the start and stop of water pump and that is represented by y; while a pair of normally open contacts in the contactor serves as memory function and that is in the form of $y 1$ represented by Pt- 1 . Here follows that:

$$
y=b\left(a+y_{1}\right)
$$

The working process is as follows: If the water level is below level b, well is empty, the coils of AC contactor will be de-energized and the pump stops working. With water unceasingly gushing in the shaft, the water level is gradually higher than level $b$, contact $b$ will close, but contact $a$ is still disconnected, the pump does not run. When water reaches to level a, contact a will close; contactor coil y conducts and drives its main contact and vice contact y1 closing, the pump begins working. With the pump running, water will be drained and water level will fall. When the water level is below level a, contact a will be disconnected. Meanwhile, control circuit conducts through the vice contact $y 1$ and the water pump is still at work. Contact $b$ will not be disconnected until the water level falls below the level $b$. Then the electricity of coil $y$ is cut off, main contact disconnects and the pump stops working. [3]

Based on the principle of double logic control, circuit control chart is illustrated here which is the foundation of the automatic drainage system. As figure 2 shows,

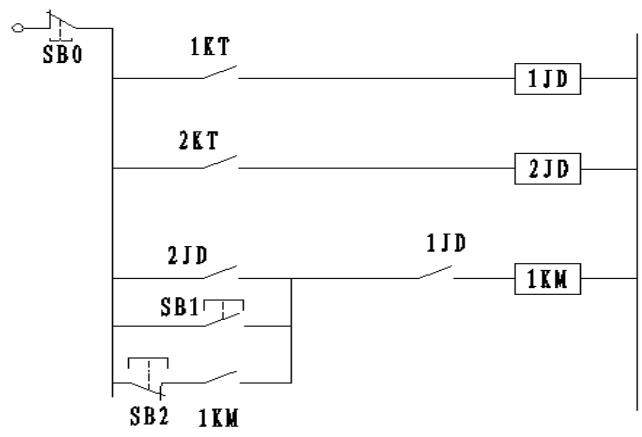

Figure 2. principle of the double control circuit

\section{The Composition And Function Of The MONITORING SYSTEM}

The double logic control system is the most basic working mode to the drainage pumping station. With the application of logic relationship between the high and low water level and pump running state, it controls the next operation of water pump along with the change of water level. In practical work, the water pump will change the control method according to various operational parameters (such as the amount of water inflow, pump condition, etc.), field environment and accidents, etc. In the drainage system of centrifugal pumps, additional work will be needed, which will make the drainage system much more complicated. [4]

The drainage monitoring system is the combination of PLC as controller, ultrasound level sensor monitoring water level, the corresponding control circuits and controlled valves and contactor as executive pieces to monitor the control system in pump station [5]. The composition of monitoring system is displayed in figure 3 .

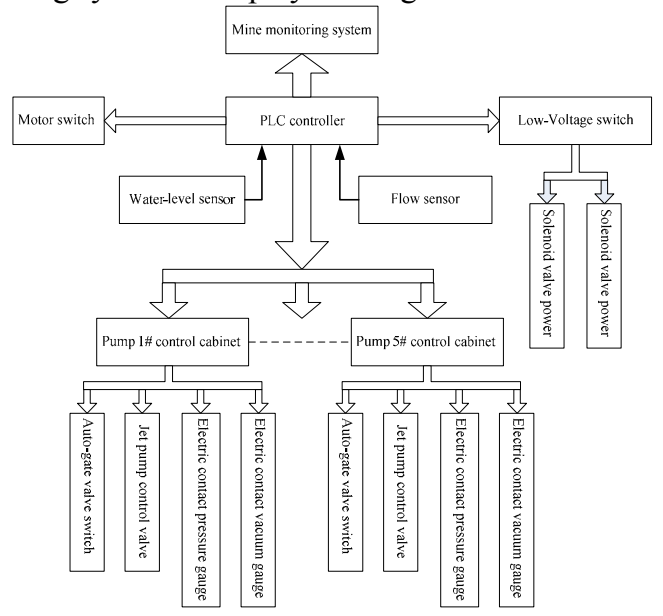

Figure 3. The structure of monitoring system

The monitoring system has three working styles, which are automatic, semi-automatic and manual. The automatic mode means that the ultrasound level sensor continually detects the variation of water level in water warehouse, and automatically opens or stops the pump and valve according 
to the water level. Normally, each pump can automatically work in rotation under the control of double logic control and "shift work system"; [2] When the water level varies too greatly, the necessary pumps will be automatically put in operation and this mode can be realized unattended. The semi-automatic mode means operating workers manually open or stop the water pump and decide the number of working pumps and motors according to water level displayed in warehouse, while the valve is, whether it is to open or close, automatically determined by PLC. The manual mode means that maintenance workers can operate any switch of pump motor, automatic valve or solenoid valve to remove mutual atresia relations.

The monitoring system can be classified into six parts: the automatic injection of water (vacuum), the manipulation of valve, the automatic monitoring of water level, the transmission of parameters, the maintenance of breakdown and the automatic control of motor. Fig. 4 shows the water drain pipes in pumping house.

1)The automatic injection of water (vacuum)

Only when the pump impellers completely submerge in the water, can the vacuum in the pump reach certain degree to realize drainage. If the vacuum is insufficient and air still exists in pump, it will result in no water pumping up and working machine "heating without water circulation", etc. The system uses jet pump to extract air, vacuum gauge to monitor vacuum degree and flow as the back-up of monitoring. It also controls the starts and stops of jet pump through the static pressure of conduit switch (control valves 10\#, 20\#, 30\#, 40\# and 50\#).

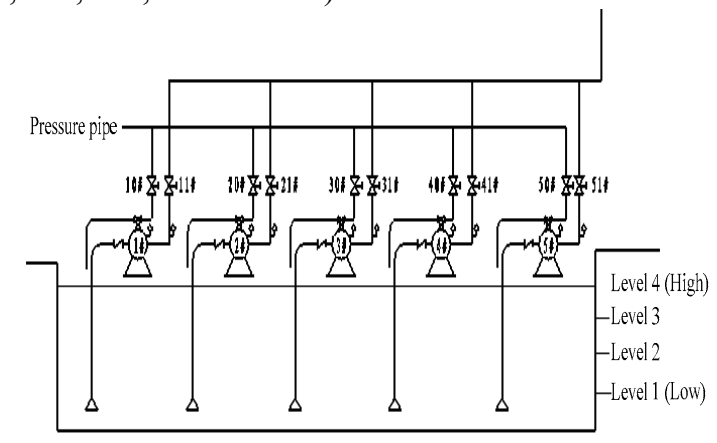

Figure 4. Pipelines in pump housing

2)The manipulation of controllable valves

To reduce the start-up power, the operation procedures of water pump require the outlet valve to be turned off when starting the centrifugal pump. When stopping the pump, in order to avoid water hammer accident, first close the valve to gradually reduce to the speed of flow and finally stop the pump.

Take the pump 1 \# as an example. After the pump body filled with water, the process is as follows. Open control valve $10 \#$, vacuuming; when the vacuum degree meets the requirements, start the motor 1 \#. After the outlet pressure reaches the set value, open the valve 11 \# for drainage and close the valve $10 \#$. When it stops, turn off the valve 11 \# and then stop the pump motor 1 \#. 3)the automatic monitoring of water level

The task of the automatic monitoring is to automatically drive or stop pumps according to the water levels. Based on the principle of double logic control, collect water-level signals and transmit them to PLC in the form of the switching value (water level switch) and analogue quantity. The reliability and accuracy of water-level sensor directly affects that of the whole control system. Therefore, ultrasonic level sensor is preferred with its own advantages, such as high accuracy, non-contact, non-mechanical type, convenient maintenance, easy installation, simple calibration, etc.

When the water level reaches the level 2, start pump if it is at low billing period of electricity and postpone starting if it is at a high billing period; when the water level rises to the level 3, start pump immediately, no matter it is in low or high billing period. If the water level climbs to the level 4 , indicating that one water pump is not sufficient to exclude mine water gushing, start the second pump, with two water pumps draining, at its maximum drainage capacity. No matter how many pumps are operated, the water level must be below the level 1 before they stop draining. The information of water level changing from 1 to 4 will be sent to PLC by the ultrasonic liquid gauge in the form of analog signals and calibrated by PLC via software. Time-sharing billing will also be calibrated by PLC via software.

4)the transmission of parameters

The analog screen of the main control cabinet can indicate water level, water flow, pump pressure and the working states of motor, solenoid valve and automatic valve. All parameters and working states will be transmitted to computer above ground by PLC. After analyzing, the information will be displayed on the analog screen in the form of curves and reports so that the managers above ground can make judgments and send commands to PLC.

5)the maintenance of breakdowns

(1).Flow protection: when the pump starts working or it is in normal condition, if the water flow can not reach the normal value, stop the current pump and start another through the flow protection device;

(2) .Motor breakdown: PLC monitors breakdowns of pump motor, such as under voltage, over current and short circuit. High-voltage switchgear will provide comprehensive protection and participate in control;

(3) The normality of the valve is to be judged through the measurement of the parameters such as the pressure, flow and pipeline of the valve in operation, the result of which is to be fed back to PLC so that the breakdown could be protected and controlled.

6)the automatic control of motor

It is the key point of the automatic drainage system, consisting of PLC, intermediate relay and the contactor. The previous five parts should be in accord with the final one. The motor will automatically open or stop the pump, and decide how many pumps should be put in operation according to the change of water level.

In case of dampened motor for long time being unused or some hidden breakdowns so that it might not be put into 
operation in emergency, "shift work system" is to be adopted in this part in order to guarantee the safety with an early discovery and treatment of the breakdown. Based on the starting number of pumps, the system will automatically operate pumps in rotation. When one pump or its subordinate valve fails to work or is in maintenance, this pump should be excluded and the rest continue draining in rotation.

\section{SUMMARIES}

1)The monitoring system adopts on-line monitoring to the equipment operation in the water pump room, and with its self-diagnosis function, unmanned operation can be achieved in the pump room. Then it will exchange data through the network and mine monitoring system and accept instructions from managers.

2)The system can realize shift between the various control modes to be complied with different working conditions. Normally, it automatically operates motors according to the water level; during breakdown or maintenance, the system can operate in semi-automatic or manual mode, that is, it can "truncate" the ongoing operation.

3)According to the water level control principle, pump shift can be automatically realized in rotation to avoid the same pump working for too long time while other pumps lying idle. Different drainage scheme will be chosen according to the water gushing conditions. In this way such cases can be avoided: when water gushing is small, pumps frequently start, or when water gushing is too large, water can not be promptly discharged. Thus, the working life of pumps can be greatly prolonged.

4)In order to improve the quality of power grid, mine monitoring system can determine the start or stop of pumps according to the peak load shifting of the power grid load.

\section{REFERENCES}

[1] Zhang Kai, Liang Jianmin, Zhang Shuguo. The optimizing of mine drainage system [J]. Coal Science and Technology. 1996. 24(1): 3132

[2] Li Fugu. Economic operation of coal mine main pumping equipment [J]. Coal Mine Machinery. 2003. 3(6): pp.94-96

[3] Zhang Jingcheng, Zhang Liqiu. Water pump and water pumping station [M]. Harbin: Harbin Institute of Technology Press. 2003

[4] Liu Bing, Chen Hongliang, Jia Lixin. Design of Automatic Monitoring System for Mine Water Pump [J]. Colliery Mechanical \& Electrical Technology. 2004. 7(5): 100-102

[5] Wang Xiaoying, Zhang Fengmin, Zhang Xuesong. The Application of PLC in mine drainage system [J]. China Coal. 2002. 28(8): 45-48 